\title{
Comparative Study of McDonald's and Subway: A Customer View of Marketing Communication Mix and Consumer Preference
}

\author{
Monica Sonkusare \\ Pandit Deendayal Petroleum University
}

\begin{abstract}
The dynamism of the food industry in India brings with itself a majority of consumers bent towards home-cooked foods. This potentially could have posed as a major challenge to major fast food retailers that ventured in the Indian market-space to experiment and establish their respective business mantras. But, despite being subjected to rapid changes in socio-economic demography of the country, the fast food industry could estimate and eventually exploited the underlying possibilities for accelerated growth and franchise development. This research revolves around the observations of a survey which was conducted to put specific focus on students of age group 18-22 block of the age group in the Ahmedabad and Gandhinagar regions of Gujarat, India. The survey witnessed 293 respondents answering the questionnaire. It entails the comparative analysis of consumer preference in two major international QSR (quick service restaurant) chains - Subway and McDonald's. Owing to the wide popularity and the fierce rivalry on the ground between McDonald's and Subway, the surveyee were receptive and responsive to the questions put forth. Cross-sectional Tabular Analysis was conducted to analyze the survey. Keeping in mind the dynamic preferences towards food traditions and ethos, this conceptual study works solely on the facts and popular opinions. As an additive, the marketing strategies and their psychological effect on the mindset of the consumer are also analyzed in this paper. Primarily, this study aims to examine the QSR differentiation in the minds of consumers, customers and non-customers.
\end{abstract}

Keywords: Consumer preference; Market Communication Mix; McDonald's; QSR; Subway 